\title{
L'Atlas de l'Estrie : un système d'information géographique régional sur Internet
}

\author{
Bérengère Ruellan ${ }^{1,2}$, David Bachy ${ }^{2}$, Claude Caron ${ }^{2}$, \\ Jean-Marie Dubois ${ }^{3}$, Marcel Laperle ${ }^{3}$ \\ Université de Sherbrooke
}

\section{Introduction}

Connaitre un territoire est un préalable fondamental à toute possibilité de pouvoir y vivre, $\mathrm{y}$ interagir et y évoluer convenablement. De même, cette connaissance permet de façonner ce territoire et de le faire évoluer vers des configurations apportant un mieux-être collectif. Dans cet esprit, un atlas régional constitue un outil de connaissance précieux permettant aux utilisateurs de découvrir les particularités de leur « coin de pays ».

C'est suivant cette volonté de se doter d'un outil régional de gestion et de diffusion des connaissances territoriales qu'a été mis en cuvre le projet de l'Atlas de l'Estrie. Réalisé sous l'égide de l'Atlas du Québec et de ses régions, ce projet a impliqué de nombreuses personnes depuis plusieurs années déjà, et le résultat est maintenant prêt à être mis à la disposition de la population estrienne.

Cet article vise à présenter l'origine de cet atlas, son contenu et son état actuel. Sont surtout mises en relief les particularités dynamiques et en ligne de cet atlas. Finalement, les perspectives futures de son développement sont esquissées.

\section{D’où vient l'idée d'atlas ?}

Le projet d'Atlas du Québec et de ses régions et d'Atlas de l'Estrie n'est pas issu d'une génération spontanée. Ce projet a une longue histoire commencée il y a plus de quarante ans, avec le début de la prise de conscience des géographes québécois de leur identité et de leur territoire. Cette histoire est aussi intimement liée au développement des moyens d'expression et de communication.

\section{Le cheminement de l'idée d'un atlas au Québec}

On pourrait dire que le rêve d'un atlas du Québec remonte à la publication, en 1958, de l'Atlas du Canada et des premières rencontres scientifiques des géographes au Québec la même année. Le projet germait dans l'idée de plusieurs fondateurs de l'Association des géographes du Québec, en 1962. Régulièrement, de la fin des années 1960 au milieu des années 1980, il était question de ce projet lors de colloques portant sur la cartographie, sur la "géographie nationale », sur l'organisation du territoire ou sur l'aménagement. Mais un tel projet n'a jamais vu le jour faute d'une équipe décidée et de moyens financiers puisque, à l'époque, la cartographie était manuelle et les coûts de l'impression en couleurs démesurés.

Par contre, un des éléments déclencheurs du projet d'Atlas du Québec et de ses régions est certainement la publication, en 1995, du premier tome de l'Atlas historique du Québec par une équipe de l'Université Laval; mais c'était encore un atlas dans le style classique en format papier.

La production d'atlas en format papier est longtemps demeurée un problème étant donné le coût élevé de production et d'achat. De plus, aussitôt publié, ce type d'atlas est désuet dans notre monde où l'appropriation du territoire se modifie de plus en plus rapidement et où la démographie s'accélère. La possibilité de remplacer le papier par la consultation électronique au milieu des années 1990 ainsi que de pouvoir produire 
des cartes polychromes au même prix que des cartes monochromes a vraiment été le principal élément déclencheur de l'Atlas du Québec et de ses régions chez ses promoteurs, d'autant plus qu'on pouvait ainsi actualiser l'information à volonté et à faible coût.

\section{Le projet de l’UQÀM pour le Québec}

L'idée d'un atlas du Québec informatisé vient d'une équipe du Département de géographie de l'Université du Québec à Montréal (UQÀM), en 1992. À l'époque, on visait encore un atlas en format papier. Deux ans plus tard, les animateurs de ce projet ont vite compris que, compte tenu de la tâche, ils devaient faire appel à l'expertise des collègues des autres institutions et des autres régions. Ils présentèrent donc leur projet lors d'un premier colloque dans le cadre du congrès de l'ACFAS à Chicoutimi, en 1995, et recueillirent ainsi les idées de la communauté universitaire sur la forme et le contenu du futur atlas. Il était évident pour tous que cet atlas devait être électronique, mais on se demandait encore à l'époque s'il ne devait pas y avoir une contrepartie en format papier ${ }^{4}$. Par contre, il était évident qu'il fallait produire un ouvrage dynamique tant dans sa forme que dans son contenu pour éviter qu'il ne devienne rapidement désuet.

Vers la fin de 1996, le projet tripartite d'atlas national, d'atlas interrégional et d'atlas régionaux prit forme et des comités interinstitutionnels de direction scientifique et de rédaction cartographique furent mis en place ${ }^{5}$. Cette concertation suscita un intérêt manifeste des collègues universitaires et stimula la recherche dans plusieurs universités du Québec.

\section{Les atlas régionaux et l'Atlas de l'Estrie}

Même stimulés par l'équipe de l'UQÀM, les atlas régionaux demeurent une initiative et une organisation des régions elles-mêmes. L'objectif est de produire des atlas régionaux qui rencontrent les besoins, les réalités et les problématiques régionales. Ces atlas peuvent donc avoir des contenus distincts les uns des autres en fonction des vues régionales et des caractères distinctifs des régions.

Le projet de l'Atlas de l'Estrie a commencé en 1999 avec un financement de l'Université de Sherbrooke et du ministère des Régions, ce qui a permis de développer la méthodologie de création des cartes et des

autres pages de l'atlas ainsi que la structure électronique de base ${ }^{6}$. En 1999 et en 2000, la méthodologie a été mise à l'essai dans le cadre d'un cours de cartographie thématique et expérimentale. Cette formule a été poursuivie depuis 2002, mais bonifiée par la participation d'un comité externe formé de trois enseignants du Département de géographie et télédétection.

\section{Vers la fin de 1996, le projet tripartite d'atlas national, d'atlas interrégional et d'atlas régionaux prit forme et des comités interinstitutionnels de direction scientifique et de rédaction cartographique furent mis en place.}

Une première version fonctionnelle interne de l'Atlas de l'Estrie a été produite à l'été 2003 grâce à un projet d'emploi étudiant, et le contenu a été augmenté au début de 2004. Le début tardif et la lenteur des travaux de cet atlas, par rapport au projet global, n'est pas dû à un manque d'intérêt des intervenants mais plutôt à un manque de financement régional. La mise en place, en 2003, du Groupe GéoBusiness (géomatique d'affaires) à la Faculté d'administration de l'Université de Sherbrooke est venue stimuler le projet.

\section{L'Atlas de l'Estrie}

Selon le plan original, l'objectif de l'Atlas de l'Estrie est d'élaborer un outil :

- de développement régional en facilitant la prise de décision des gestionnaires;

- de formation du citoyen par le biais de la vulgarisation et de l'approfondissement de la connaissance du milieu régional;

- de formation académique et professionnelle à tous les niveaux de formation;

- de promotion régionale à l'extérieur de la région;

- de conservation de la mémoire collective régionale.

Chaque thème de l'Atlas doit donc avoir un contenu qui s'adresse aux publics suivants :

- citoyens en général : sensibilisation et promotion;

- gestionnaires;

- spécialistes;

- étudiants à tous les niveaux. 
Le territoire concerné par l'Atlas de l'Estrie comprend tout ce qui est perçu du point de vue estrien. Ainsi, les thèmes biophysiques couvrent tout le plateau appalachien du sud-ouest du Québec. Les thèmes socio-économiques couvrent les sept MRC de la région administrative de l'Estrie : Le Granit, Asbestos, Haut-Saint-François, Val-Saint-François, Sherbrooke, Coaticook et Memphrémagog. Les thèmes du tourisme et des loisirs couvrent la région touristique de l'Estrie, ce qui inclut la région administrative plus deux MRC: Brome-Missisquoi et de la HauteYamaska.

Chaque thème constituant l'Atlas est illustré par une page d'accueil contenant généralement une carte synthèse couvrant l'Estrie au complet et des pages connexes. Les pages connexes, accessibles à partir de la page d'accueil du thème, sont soit des cartes d'une MRC en particulier, soit un texte explicatif, un graphique représentant des statistiques ou une image représentative du thème. Chaque thème est présenté d'une façon originale afin d'illustrer un point de vue particulier. Le vert sert de tonalité de base pour l'ensemble du site.

Une page d'accueil introduit les visiteurs au site. Ils ont alors le choix de naviguer vers la section des systèmes biophysiques ou vers celle des systèmes humains et sociaux. Une page d'options présente quatre cases déroulantes. Le lecteur peut choisir l'un des thèmes du projet :

- biophysique : géologie, hydrographie, foresterie et climatologie;

- socio-économique : transports, tourisme et loisir, démographie et culture.

\section{Chaque thème constituant l'Atlas est illustré par une page d'accueil contenant généralement une carte synthèse couvrant I’Estrie au complet et des pages connexes.}

À ces thèmes s'ajoutent d'autres thématiques présentées sous forme de cartes dynamiques, c'est-à-dire dont les variables (zone choisie, année, variable socio-économique, etc.) peuvent être paramétrées en temps réel par l'utilisateur de l'Atlas. Selon ce principe et suivant une approche de type hyper-atlas, les thèmes régionaux de l'Atlas de l'Estrie sont présentés non pas d'une façon statique et de l'extérieur, mais selon le vécu des Estriens et en présentant la dynamique associée à chaque thème. Chaque thème est présenté davantage selon un mode explicatif que descriptif. Pour cela, chaque carte est accompagnée de pages explicatives de type texte, tableau, graphique ou photo. Le lecteur qui désire une vue générale et superficielle ne regardera que les cartes. Par contre, le lecteur intéressé à approfondir le thème consultera les autres types de pages associées.

\section{L'apport des sites dynamiques}

De manière à en arriver à un atlas comportant un contenu à la fois très complet et interactif, il a été décidé d'ajouter au site les possibilités apportées par les dernières technologies de pointe en matière de publication de données géographiques sur Internet. Pour constituer un atlas dynamique en ligne, deux types de dynamisme sont possibles : un dynamisme en ce qui concerne la carte et un dynamisme en ce qui a trait à l'interface.

\section{Carte dynamique}

Une carte dynamique sur Internet offre de nombreuses fonctions permettant de naviguer dans cette carte et d'y consulter les données géographiques. Ainsi, l'utilisateur a la possibilité de faire différentes manipulations sur la carte comme naviguer (zoom avant, zoom arrière, déplacement, etc.), gérer les couches ou thèmes d'information affichés (afficher ou non, changer les symboles utilisés, etc.) ou consulter les données (poser des questions relativement aux données de base constituant les cartes, effectuer des requêtes spécifiques à la base de données, etc.). Une telle carte dynamique en ligne (appelé Web-Based GIS dans le jargon du métier) est en réalité un type particulier de système d'information géographique permettant de consulter de façon interactive les données géographiques sur Internet.

Dans le cas de l'Atlas de l'Estrie, les cartes dynamiques ont été réalisées dans le cadre d'un stage de fin d'études du premier auteur, de même que la collecte de données géographiques de la région de l'Estrie et leur intégration dans le système d'information géographique sur Internet. 
Les données de la carte topographique dynamique sont issues de la BNDT (Base nationale de données topographiques) de Géomatique Canada. Ceci comprend les éléments habituellement représentés sur une carte topographique aux échelles du $1 / 50000^{\mathrm{e}}$ et du $1 / 250000^{\mathrm{e}}$ : voies de communication, cours d'eau et lacs, relief, bâtiments, etc. Par contre, pour ce qui est des autres cartes thématiques, les données géométriques et descriptives proviennent de Statistique Canada, et plus particulièrement des recensements nationaux de 1996 et 2001. Ces données permettent de comparer les données dans le temps et dans l'espace. Par exemple, il est possible de connaître la population par MRC de l'Estrie en 1996 et en 2001, mais aussi par municipalité pour ces deux dates.

Pour pouvoir publier ces données géographiques dans Internet, nous avons utilisé le logiciel JMAP de la compagnie Kheops Technologies. Ce système possède de nombreuses fonctions facilitant la production des cartes dans Internet telles la sélection des thèmes à afficher, les symboles à utiliser, l'échelle d'affichage, les paramètres techniques des cartes comme la projection et les étiquettes de texte. Une fois ces paramètres spécifiés, la carte est prête à être publiée dans Internet par JMAP selon un gabarit de carte prédéfini (fig. 1).

\section{Figure 1 - Carte topographique dynamique}

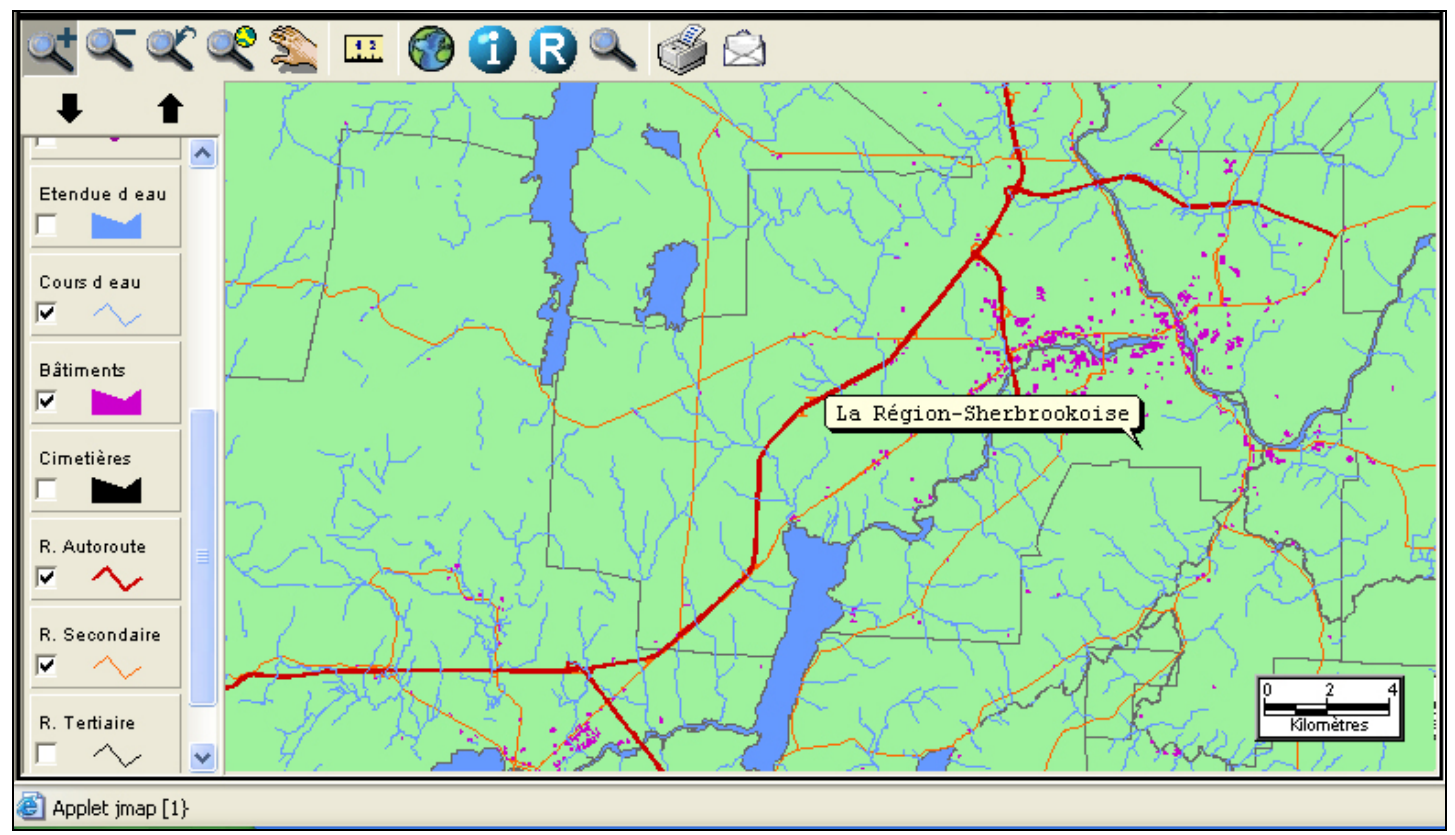

\section{Interface dynamique}

Un atlas en ligne constitué d'une interface dynamique présente de nombreuses fonctions facilitant l'interaction de l'internaute avec l'atlas. Ainsi, un menu dynamique permet d'afficher les différents thèmes que l'utilisateur peut consulter. En plus de ces menus dynamiques, un ensemble de listes déroulantes et de cases à cocher permettent à l'utilisateur de pouvoir préciser les spécifications de sa carte thématique (par exemple, la partie de territoire concernée, la période à analyser ou le thème à illustrer).

La figure 2 illustre une carte thématique ayant une interface de type dynamique. L'utilisateur choisit d'a- bord le thème qu'il souhaite consulter (les deux menus en haut de la page : milieux biophysiques et milieux humains et sociaux). Ensuite, l'utilisateur choisit le type de carte thématique en fonction des choix proposés par l'atlas en ligne. Par exemple, la figure 2 montre une carte thématique de la répartition de la population dans les municipalités de la MRC de Sherbrooke en 2001. Cette interface dynamique propose aussi à l'utilisateur de choisir d'autres thématiques concernant la population, comme l'évolution démographique sur différents types de territoire (par MRC ou par municipalité) et pour une certaine année (ici, 1996 ou 2001).

Dans ce cas, il s'agit de cartes statiques, c'est-à-dire entièrement prédéfinies. Par contre, le dynamisme de 
l'interface permet d'aiguillonner le serveur de cartes pour qu'il transmette dynamiquement les cartes prédé- finies. Ces cartes sont ainsi appropriées en fonction des paramètres définis par l'utilisateur.

Figure 2 - Carte thématique avec interface dynamique

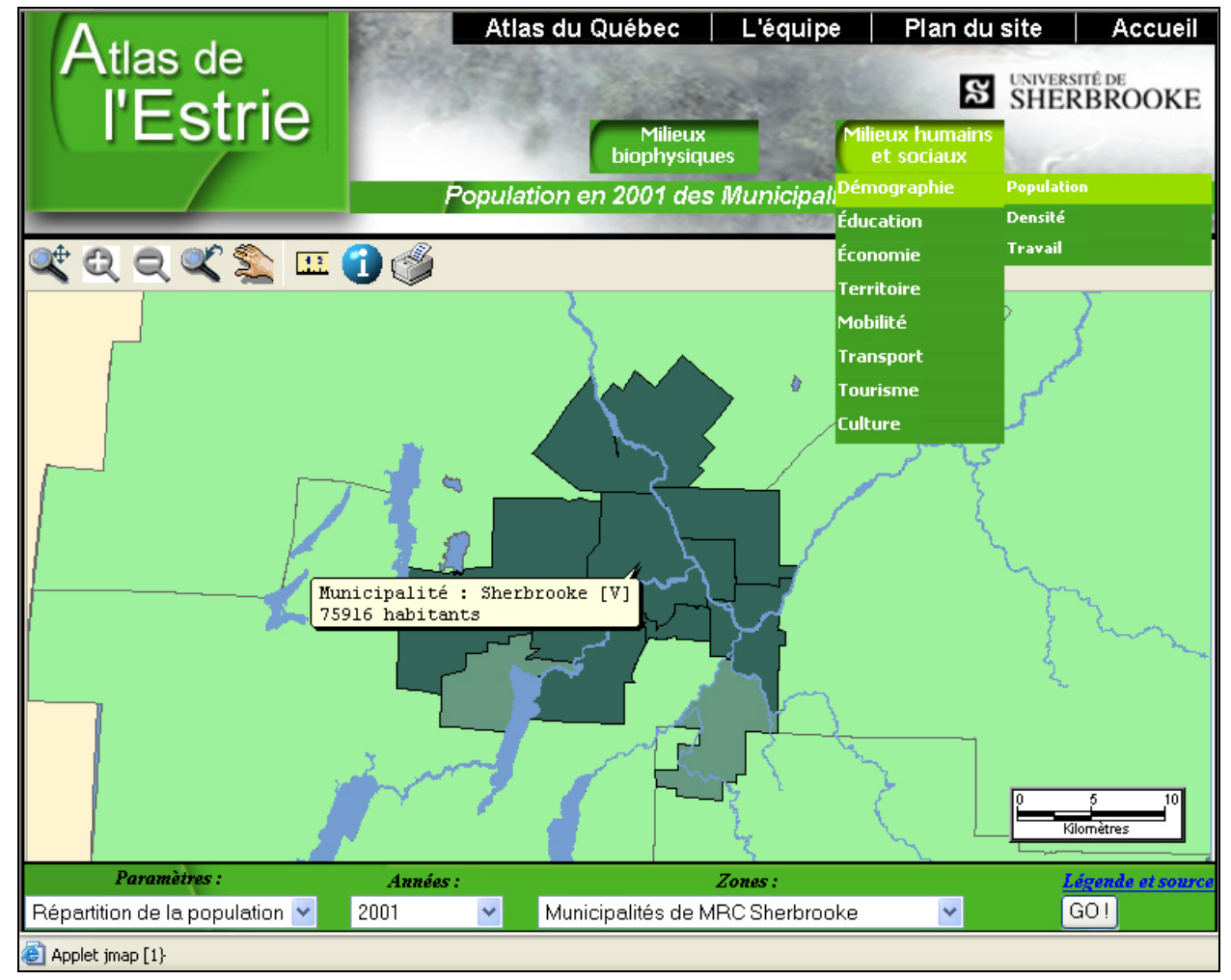

\section{Les perspectives de développement}

L'Atlas de l'Estrie est maintenant rendu à un niveau d'avancement suffisant pour qu'il soit mis à la disposition de la population estrienne. Il constitue déjà un outil informationnel, et donc d'aide à la prise de décision, très intéressant et efficace. Dans le but de le parfaire, il reste encore certaines étapes de réflexion et de développement devant être menées à bien, à savoir :

- s'assurer que le choix des thèmes disponibles est le plus pertinent possible pour les utilisateurs de l'Atlas; en ce sens, il sera souhaitable d'interagir avec les utilisateurs pour recueillir leurs commentaires et suggestions;

- ajouter des thèmes complémentaires de manière à enrichir le contenu de l'Atlas;

- revisiter les thèmes déjà en ligne de manière à les peaufiner et à les compléter; il s'agira notamment d'ajouter des données temporelles au fur et à mesure que les données statistiques seront disponibles;
- améliorer l'ergonomie de l'interface à l'utilisateur; il s'agira aussi de simplifier les fonctionnalités de navigation cartographique existantes et d'ajouter de nouvelles fonctions d'affichage et d'interrogation;

- accélérer le temps de transfert des données entre le serveur et l'ordinateur des utilisateurs; en effet, certaines cartes s'avèrent un peu longue à afficher lorsque l'utilisateur ne bénéficie pas d'une large bande passante sur Internet.

- envisager la possibilité que certains thèmes soient standardisés dans les atlas national, interrégional et régionaux pour des fins de comparaison et de réflexion sur les limites territoriales naturelles et construites.

L'Atlas de l'Estrie constitue déjà un outil précieux pour connaître le territoire estrien. Il peut être consulté à l'adresse : http://www.atlasduquebec.qc.ca, en choisissant la section Les atlas régionaux, puis Estrie. Il s'agira maintenant que les utilisateurs s'approprient 
ce nouvel outil et proposent des façons de l'enrichir et de l'améliorer. Ceci permettra d'en arriver à un système d'information qui répondra encore mieux aux préoccupations des gens qui habitent ce territoire. En ce sens, les auteurs du présent article sont ouverts aux suggestions qui permettront d'améliorer «votre» atlas.

\section{Notes et références}

1 Licence professionnelle des Systèmes d'Information Géographique, Université de La Rochelle (France), Faculté des Lettres, Langues, Art et Sciences Humaines.

2 Groupe GéoBusiness, Université de Sherbrooke, Faculté d'administration.
3 Université de Sherbrooke, Faculté des lettres et sciences humaines, Département de géographie et télédétection.

4 Dubois, J.-M.M. (1995). « Un atlas du Québec basé sur les dynamiques de forme et de contenu ", $63^{e}$ Congrès de l'ACFAS, 22-26 mai, Chicoutimi, recueil des résumés de communications, vol. 63, p. 500 .

5 Carrière, J. et, J.-L. Klein (2003). «L'atlas du Québec et de ses régions : un inventaire du patrimoine territorial québécois sur l'autoroute de l'information », Geomatica, vol. $57, \mathrm{n}^{\circ} 2$, p. $177-181$.

6 Rodrigue, J.-F. (2001). « Méthodes appliquées à la création du site Web de l'Atlas de l'Estrie », rapport de baccalauréat, Université de Sherbrooke, Département de géographie et télédétection, $107 \mathrm{p}$. 